\title{
Retrospective analysis of bacterial colonization of necrotic bone and antibiotic resistance in 98 patients with medication-related osteonecrosis of the jaw (MRONJ)
}

\author{
Florian Ewald ${ }^{1} \cdot$ Falk Wuesthoff $^{2} \cdot$ Robert Koehnke $^{2} \cdot$ Reinhard E. Friedrich $^{2} \cdot$ Martin Gosau $^{2} \cdot$ Ralf Smeets $^{3} \cdot$ \\ Holger Rohde ${ }^{4}$. Alexandre T. Assaf ${ }^{2}$ (D)
}

Received: 29 May 2020 / Accepted: 15 September 2020 / Published online: 2 October 2020

(C) The Author(s) 2020

\begin{abstract}
Objectives The aim of our study was to describe microbial flora associated with MRONJ and characterize the susceptibility of pathogens to help guide an effective empiric antibiotic treatment in these patients.

Materials and methods A retrospective, single-center analysis was performed, using 116 bone samples from 98 patients. The bone samples were homogenized and subjected to routine culture methods. Growing bacteria were differentiated to the species level using whole-cell mass spectrometry and subjected to susceptibility testing.

Results A highly diverse microbial flora was detected in necrotic bone, with a simultaneous presence of two or more bacterial species in $79 \%$ of all patients. In at least $65 \%$ of samples, gram-negative isolates were detected. Therefore, bacterial species resistant against $\beta$-lactamase inhibitors were present in at least $70 \%$ of all patients.

Conclusions The empiric choice of antibiotics in MRONJ patients should consider the high rate of gram-negative bacteria and resistance against $\beta$-lactam antibiotics.

Clinical relevance According to recent guidelines and recommendations, systemic antibiotic treatment is a key component in the treatment of all stage 2 and $3 \mathrm{MRONJ}$ patients. We recommend using fluoroquinolones for empiric treatment and emphasize the use of bacterial cultivation and susceptibility testing to enable an effective antibiotic treatment.
\end{abstract}

Keywords Bacterial colonization - Antibiotic resistance $\cdot$ Medication-related osteonecrosis of the jaw $\cdot$ Antiresorptive drug-induced osteonecrosis of the jaw $\cdot$ ARONJ $\cdot$ MRONJ $\cdot$ Oral microbiota

Electronic supplementary material The online version of this article (https://doi.org/10.1007/s00784-020-03595-9) contains supplementary material, which is available to authorized users.

\section{Alexandre T. Assaf}

a.assaf@uke.de

1 Department of General, Visceral and Thoracic Surgery, University Medical Center Hamburg Eppendorf, University of Hamburg, Hamburg, Germany

2 Department of Oral and Maxillofacial Surgery, University Medical Centre Hamburg Eppendorf, University of Hamburg, 20246 Hamburg, Germany

3 Department of Oral and Maxillofacial Surgery, Division of Regenerative Orofacial Medicine, University Medical Centre Hamburg Eppendorf, University of Hamburg, Hamburg, Germany

4 Department of Medical Microbiology, Virology and Hygiene, University Medical Centre Hamburg Eppendorf, University of Hamburg, Hamburg, Germany

\section{Introduction}

Antiresorptive drug induced- or medication-related osteonecrosis of the jaw (ARONJ, MRONJ) became a serious disease pattern in recent years. The number of patients receiving intravenous (e.g., zoledronate) or oral bisphosphonates (e.g., alendronate) [1] as well as subcutaneous treatment with RANKL inhibitors (e.g., denosumab) or compounds with antiangiogenic effects (i.e., bevacizumab, sorafenib, sunitinib, and others) have been rising over the last decade [2-4]. Indications for antiresorptive drug treatment are nonneoplastic diseases, such as osteoporosis [2], osteitis deformans (Paget's disease) or arthritis [5], and neoplastic diseases, such as tumor-associated hypercalcemia, multiple myeloma, and skeletal metastases from carcinomas (e.g., breast cancer, renal or prostate cancer) [6-8]. The estimated cumulative incidence of MRONJ in patients receiving bisphosphonates or RANKL inhibitors 
(e.g., denosumab), human monoclonal antibodies (e.g., bevacizumab), or protein kinase inhibitors (e.g., sorafenib/sunitinib) is thought to be between 0.4 and $21 \%$, depending on the dose and compound used, as well as the route of administration $[1,9,10]$.

The diagnostic criteria of MRONJ include an exposure history to bisphosphonates, RANKL inhibitors, or antiangiogenic drugs, exposed bone within the oral cavity, and no history of prior radiation therapy to the jaws [11]. Further affections associated with exposed intraoral necrotic areas are extraoral fistulas, resulting from necrotic bone lesions [1]. Next to a detailed intraoral examination, initial diagnostic procedures routinely include $\mathrm{X}$-ray analysis (e.g., panoramic view, cone beam computed tomography, or computed tomography scans) [12, 13], as well as magnetic resonance imaging (MRI) and scintigraphy [14].

In recent years, it became increasingly evident that bone colonization with bacteria and possibly also fungi plays an important role in the pathogenesis of MRONJ [15]. Healthy bone tissue in the maxilla and mandibula seems to be resistant to microbial colonization, even if exposed to the oral flora. However, in patients treated with antiresorptive or antiangiogenic agents, it is hypothesized that conditions creating an access for bacteria and other pathogens to the vulnerable bone can trigger the development of MRONJ. These conditions include dental procedures, periodontal disease, trauma, or poorfitting prosthetic devices. In line with this hypothesis, MRONJ predominantly occurs in regions of the body that are exposed to microbial flora like that of the oral cavity [15], whereas MRONJ rarely occurs in other, aseptic regions of the skeletal system.

Although the exact mechanisms still need to be elucidated, there are several hypotheses regarding the mechanisms by which bacterial colonization of infection could induce osteonecrosis. These include the release of acids and proteases, inhibition of bone matrix synthesis, or stimulation of bone degradation $[15,16]$. Of note, gram-negative bacteria are hypothesized to play a predominant role in the process by producing toxic products including lipopolysaccharides, directly inducing osteoclast differentiation and activity [15].

Therefore, one of the major aspects in the treatment of MRONJ patients are infections of the adjacent bone and surrounding soft tissues. Especially in MRONJ stages 2 and 3, an effective treatment of bacterial colonization and infection in the affected areas needs to be included into the treatment plan of each patient [16]. The goal of this study was to characterize the composition of colonizing bacteria related to necrotic bone lesions, thereby providing guidance in establishing an effective antibiotic treatment.

\section{Material and methods}

\section{Patient characteristics}

This study is a monocenter, retrospective study. Between June 2016 and September 2018, 98 patients treated at the University Medical Center Eppendorf for clinically and histopathologically confirmed osteonecrosis of the jaw were included in this study (Table 1). The mean age at surgery was $70.9 \pm 10.4$ years, with a slight predominance of female patients ( $n=53,54.1 \%$ of all patients). All patients had exposed bone in the oral cavity and were clinically symptomatic (osteonecrosis of the jaw stages 2 or 3 , according to the

Table 1 Demographic and clinicopathologic characteristics of study patients

\begin{tabular}{|c|c|c|}
\hline & $n$ & $\%$ \\
\hline Male & 45 & 45.9 \\
\hline Female & 53 & 54.1 \\
\hline Age at surgery & 70.9 & \\
\hline \multicolumn{3}{|l|}{ Underlying diagnosis } \\
\hline Breast cancer & 26 & 26.5 \\
\hline Prostate cancer & 22 & 22.4 \\
\hline Multiple myeloma & 16 & 16.3 \\
\hline Osteoporosis & 15 & 15.3 \\
\hline Renal cancer & 9 & 9.2 \\
\hline Lung cancer & 4 & 4.1 \\
\hline Aggressive systemic mastocytosis & 2 & 2.0 \\
\hline Other ${ }^{\S}$ & 4 & 4.1 \\
\hline \multicolumn{3}{|l|}{ MRONJ stage at diagnosis } \\
\hline Stage 2 & 88 & 89.8 \\
\hline Stage 3 & 10 & 10.2 \\
\hline \multicolumn{3}{|l|}{ Localization } \\
\hline Upper jaw & 29 & 25.0 \\
\hline Lower jaw & 75 & 64.7 \\
\hline Both & 12 & 10.3 \\
\hline \multicolumn{3}{|l|}{ Antiresorptive drug regimen } \\
\hline Bisphosphonate & 57 & 58.2 \\
\hline Denosumab & 33 & 33.7 \\
\hline $\mathrm{BP}+$ denosumab & 5 & 5.1 \\
\hline Other ${ }^{\&}$ & 3 & 3.1 \\
\hline \multicolumn{3}{|l|}{ Trigger factor for MRONJ development } \\
\hline Poor prosthesis fit & 26 & 26,5 \\
\hline Tooth extraction & 50 & 51,0 \\
\hline Unknown & 22 & 22,4 \\
\hline
\end{tabular}

$\S$ One patient had metastatic thyroid cancer, chondrosarcoma, and cancer of unknown primary, respectively. One patient was treated for myelodysplastic syndrome

\&One patient had received sunitinib, azacitidine, and sorafenib, respectively 
AAOMS position paper on MRONJ from 2014 [17, 18]). MRONJ occurred more often in the mandible than in the maxilla. Of the 98 patients, 55 had received oral or intravenous bisphosphonates, 33 had been treated with denosumab, and 5 patients had received a combination of both. Three patients had no history of antiresorptive drugs but have had intensive chemotherapy for myelodysplastic syndrome $(n=2)$ or metastasized renal cancer $(n=1)$ including antiangiogenic agents [19]. None of the patients had a history of prior radiation therapy of the head or neck region.

Of the 98 patients, 17 patients had either relapsing or recurrent disease. Surgical treatment with resection of necrotic bone with local flap coverage was done twice $(n=14)$ or three times $(n=3)$ in these patients. Therefore, a total of 116 specimens were analyzed.

\section{Treatment algorithm}

After initial evaluations, all patients with clinically and radiologically confirmed diagnosis of stage 2 or 3 MRONJ were treated with oral decontamination using chlorhexidine rinses and oral antibiotics (i.e., amoxicillin and clavulanic acid, 875/ $125 \mathrm{mg}$ twice daily, in patients with no known drug intolerances against penicillin, clindamycin $300 \mathrm{mg}$ four times daily, or moxifloxacin $400 \mathrm{mg}$ once daily) for 7 days before surgical intervention. Preoperative antibiotic treatment was done using amoxicillin and clavulanic acid in about two-thirds (61\%), and moxifloxacin in about one-third of all patients (31\%). One day prior to the surgery, all patients turned to inpatient treatment, with intravenous administration of antibiotics, except for moxifloxacin, which was continued orally.

According to the German guideline for the treatment of MRONJ, antibiotic treatment should be continued until signs of local inflammation or bacterial infection of the wound resolve $[20,21]$. Therefore, the antibiotic treatment was continued postoperatively. All patients were scheduled for reevaluation and follow up after 1,2, and 3 weeks. The antibiotic regimen was then adjusted according to the results from cultivation of bone samples and were given until suture removal, usually at days 14 to 21 after surgery.

\section{Intraoperative sample collection and preparation of microbiological cultures}

At the beginning of the surgical procedure, the mouth of the patient is scrubbed with either Betadine or, in case of intolerance, octenidin. Saliva surrounding the surgical site was continuously removed using an aspirator. In order to obtain a nonsuperficial specimen of the necrotic bone, the superficial layer of necrotic bone was removed using an ultrasonic bone cutting system. Then, a sample of necrotic bone was harvested, carefully avoiding contamination of the specimen by saliva, surrounding tissue, or contaminated medical instruments.
The bone specimens were subjected to microbiological examination using routine culture methods at the Institute for Medical Microbiology of the University Medical Center Eppendorf. In brief, tissue samples were homogenized and streaked onto Columbia blood agar, chocolate agar, and Schaedler agar (all Thermo Fisher, Bremen, Germany), and incubated at $37{ }^{\circ} \mathrm{C}$ in the presence of $5 \% \mathrm{CO}_{2}$ and anaerobic, respectively. Plates were read every $48 \mathrm{~h}$ for a total of 14 days. Growing bacteria were differentiated to the species level using whole-cell mass spectrometry (Biotyper, Bruker, Bremen, Germany). Species known to potentially carry relevant acquired resistance markers (i.e., Enterococcus spp. [vanillate demethylase complex (vanAB)], Staphylococcus aureus [mecA-gene], Enterobacteriaceae [e.g., extended spectrum Blactamase (ESBL), carbapemases], Pseudomonas aeruginosa) were subjected to susceptibility testing using a Vitek II system (Biomerieux, Marcy L'Étoile, France). For species belonging to the resident flora of the oral cavity, susceptibilities were deduced from species identification.

\section{Statistical analysis}

Data collection and analysis illustrations were done using Microsoft ${ }^{\circledR}$ Excel $^{\circledR}$ and PowerPoint ${ }^{\circledR}$ (Office $365^{\circledR}$ ) and GraphPad Prism Version 4.03.

\section{Results}

\section{Bacterial infection of bone is diverse, and simultaneous presence of different bacteria is common}

In the 116 specimens of necrotic bone, the presence of 43 different bacterial genera (a total of 259 isolates were found and, of these, 199 were identified to species level) and 6 different fungal species were detected using bacterial culture and whole-cell mass spectrometry (Fig. 1).

Cultivation revealed no bacterial growth in three cases. In $18 \%$ of cases, the presence of one bacterial of fungal species was found, whereas in $79 \%$ of cases, two or more species were detected (i.e., 2 different species were detected in 35\%, 3 in $28 \%, 4$ in $11 \%$, and 5 in 5\% of cases, respectively) (Fig. 2).

Most frequently, Streptococcus spp., Neisseria spp., Lactobacillus spp., and coagulase-negative Staphylococcus genera were detected, accounting for about $60 \%$ of all isolates (Table 2). In total, 63\% of all bacterial species detected were gram-positive (Fig. 3a). However, in $65 \%$ of all bone samples, at least one gram-negative isolate was identified, i.e., in $52 \%$ both gram-positive and gram-negative bacteria were present, and in $13 \%$, gram-negative isolates only (Fig. 3b). The presence of yeast was detected in 25 of the 116 cases (21.5\%). 
Fig. 1 Overview over bacterial species and yeasts found in necrotic bone specimen. Pie chart of all bacterial genera and yeasts detected in the 116 specimens of necrotic bone. In three cases, no microbial colonization could be detected (indicated as sterile)

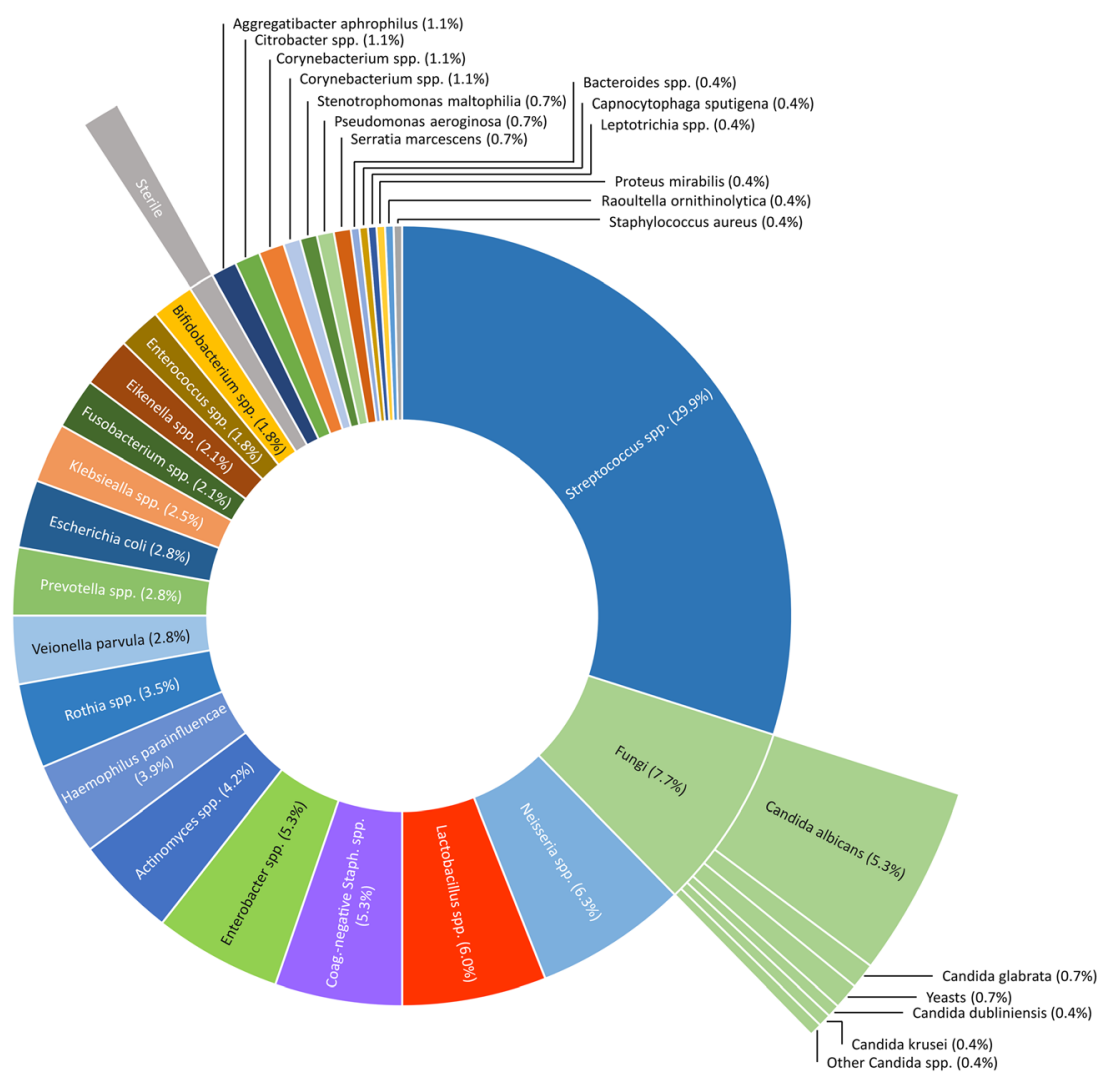

Effect of susceptibility testing on antibiotic treatment

In our study, amoxicillin and clavulanic acid were administered orally twice daily as the initial antibiotic regimen in about two-thirds of all patients (61\%) (Fig. 4b). In about one-third of patients $(31 \%)$, oral moxifloxacin was used in a once-daily regimen.

Antibiotic treatment was continued until suture removal after 14 days. Therefore, when susceptibility testing was available for 52 patients, a change of the antibiotic regiment was warranted in 31 patients. Most of these patients $(n=27)$ were treated with amoxicillin and clavulanic acid, 2 were treated with moxifloxacin, and 1 patient was treated with oral clindamycin or cefuroxime, respectively. In most cases of penicillin resistance, moxifloxacin was used as second-line therapy. As shown in Fig. 5, results from susceptibility testing influenced the antibiotic treatment of 22 patients. However, in 8 patients, no changes to the antibiotic treatment were made, either because the patient did not appear for follow-up examinations, or results from the susceptibility testing were missed. In one patient treated with moxifloxacin and the presence of resistance, the treatment was stopped. In 5 patients, the antibiotic regimen was changes for other reasons, e.g., intolerance. 


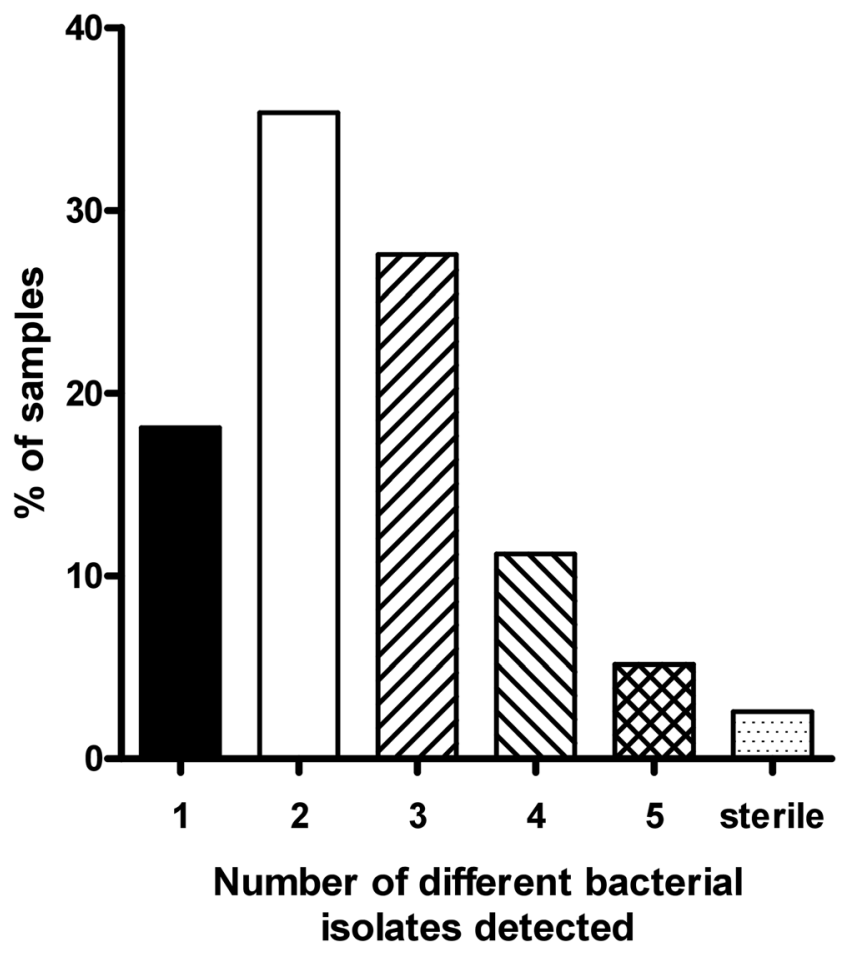

Fig. 2 Number of different bacterial species or yeasts detected in necrotic bone specimen. In $18 \%$ of the 116 cases, the presence of one bacterial of fungal species was found, whereas in $79 \%$ of cases, two or more species were detected (i.e., 2 different species were detected in 35\%, 3 in $28 \%, 4$ in $11 \%$, and 5 in $5 \%$ of cases, respectively). In three cases, no microbial colonization could be detected (indicated as sterile)

\section{Changes of the microbial composition over time in MRONJ patients treated for recurrent disease}

Seventeen patients had recurrence of MRONJ, requiring surgical intervention; three patients required surgical treatment for a third time. Therefore, 37 bone specimens were available for microbiological examination. The mean interval between surgery was 103 days, and in 15 of the 17 patients, recurrent disease involved the same location. In 9 of the 17 cases, the microbial composition found in the necrotic bone specimens differed. In 8 cases, one or two bacterial or fungal genera were present in both bone specimen. In total, of the 75 isolates found, only 9 were present in all sequential bone samples of the respective patient (Figure S1A and S1B).

\section{Discussion}

In recent treatment guidelines, there is a consensus that prolonged antibiotic treatment is indicated in MRONJ patients with signs of infection, i.e., in all patients with MRONJ stage 2 or $3[11,18,20]$.

However, there is little guidance regarding the choice of antibiotics for initial empiric therapy in these patients. To our knowledge, a comprehensive analysis of the microbiome in
Table 2 Overview over bacterial and fungal isolates found in necrotic bone specimen

\begin{tabular}{|c|c|c|}
\hline Species & $n$ & $\%$ \\
\hline Actinomyces spp. & 12 & 4.2 \\
\hline Aggregatibacter aphrophilus & 3 & 1.1 \\
\hline Bacteroides spp. & 1 & 0.4 \\
\hline Bifidobacterium spp. & 5 & 1.8 \\
\hline Capnocytophaga sputigena & 1 & 0.4 \\
\hline Citrobacter spp. & 3 & 1.1 \\
\hline Corynebacterium spp. & 3 & 1.1 \\
\hline Eikenella spp. & 6 & 2.1 \\
\hline Enterobacter spp. & 15 & 5.3 \\
\hline Enterococcus spp. & 5 & 1.8 \\
\hline Escherichia coli & 8 & 2.8 \\
\hline Fusobacterium spp. & 6 & 2.1 \\
\hline Haemophilus parainfluencae & 11 & 3.9 \\
\hline Klebsiealla spp. & 7 & 2.5 \\
\hline Lactobacillus spp. & 17 & 6.0 \\
\hline Leptotrichia spp. & 1 & 0.4 \\
\hline Neisseria spp. & 18 & 6.3 \\
\hline Prevotella spp. & 8 & 2.8 \\
\hline Proteus mirabilis & 1 & 0.4 \\
\hline Pseudomonas aeroginosa & 2 & 0.7 \\
\hline Oral flora, not specified & 2 & 0.7 \\
\hline Raoultella ornithinolytica & 1 & 0.4 \\
\hline Rothia spp. & 10 & 3.5 \\
\hline Serratia marcescens & 2 & 0.7 \\
\hline Staphylococcus aureus & 1 & 0.4 \\
\hline Staphylococcus spp. coagulase-negative & 15 & 5.3 \\
\hline Stenotrophomonas maltophilia & 2 & 0.7 \\
\hline Streptococcus spp. & 85 & 29.9 \\
\hline Veionella parvula & 8 & 2.8 \\
\hline \multicolumn{3}{|l|}{ Yeasts } \\
\hline Candida albicans & 15 & \\
\hline Candida spp. & 1 & \\
\hline Candida glabrata & 2 & \\
\hline Candida dubliniensis & 1 & \\
\hline Candida krusei & 1 & \\
\hline Other yeasts & 2 & \\
\hline No bacterial colonization detected ("sterile") & 3 & \\
\hline
\end{tabular}

necrotic bone considering susceptibility testing and antibiotic resistance has not been done yet.

To determine the spectrum of bacteria found in necrotic bone, a deep biopsy of affected bone was obtained, and routine culture techniques were applied. Other approaches, e.g., the detection of ribosomal RNA, may offer a more comprehensive coverage of bacteria present in tissues from polymicrobial infections. However, the culture method was chosen to determine the viable flora in the necrotic bone, and to allow for susceptibility testing. 
Fig. 3 Distribution of gramnegative and gram-positives isolates. $63 \%$ of all isolates were gram-positive (a). However, at least one gram-negative isolate was detected in $65 \%$ of all samples analyzed (b)

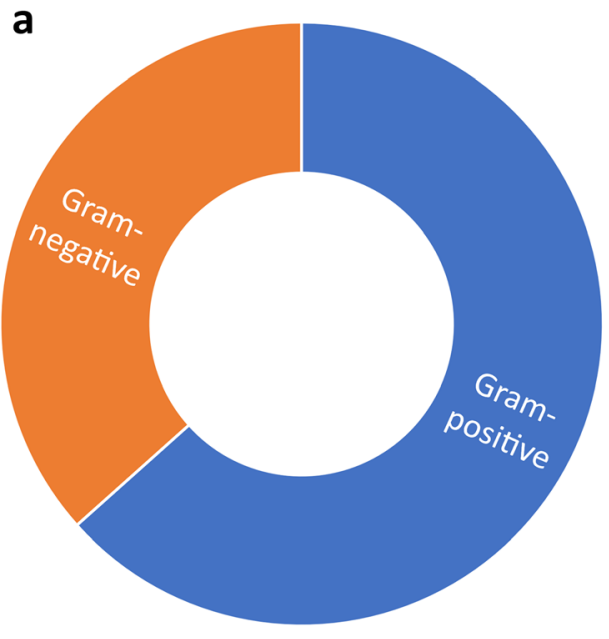

Gram-positive:

$63.4 \%$ Gram-negative:

$36.6 \%$

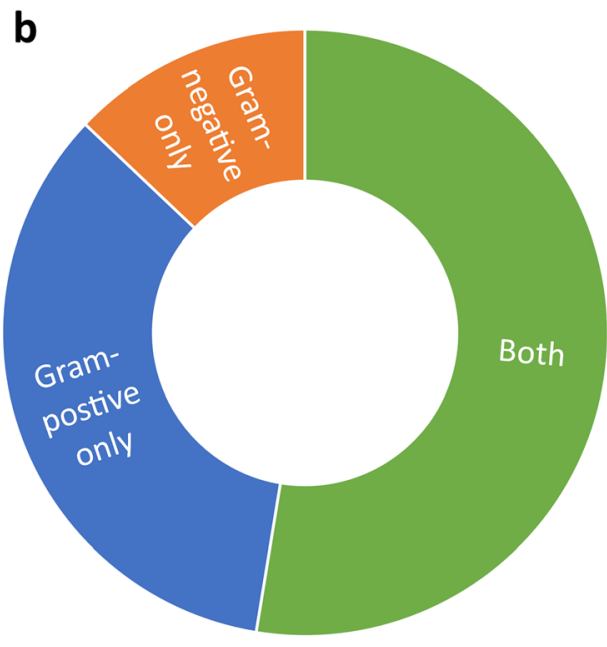

Gram-negative only:

$12.9 \%$ Gram-positive only: $34.5 \%$ Gram $^{-}$and Gram $^{+}$:
During the process of harvesting the bone samples, considerable effort was done to avoid contamination. However, we cannot rule out that minimal contamination for example by aerosol formation or medical instruments contaminated by saliva from the adjacent oral cavity may have occurred. Furthermore, handling of the bone biopsy may have affected the detection of certain bacterial species, e.g., anaerobic bacteria, and the culture methods applied may be unsuited for the detection of some species.

Nevertheless, when comparing the microbial flora in biofilms from affected bone in MRONJ patients described by de Bruyn et al. using rRNA profiling with our results, there is a considerable similarity to the microbial flora found in our study. This includes the detection of bacterial genus associated with MRONJ development like Aggregatibacter actinomycetemcomitans, Prevotella spp., Fusobacterium or Capnocytophaga species, Streptococcus mitis, Streptococcus gordonii, Actinomyces odontolyticus, and Veillonella species [15, 16, 22].

In previous studies on the microbiome in MRONJ patients, an important role of Actinomyces species in the establishment of biofilms has been proposed. Actinomyces species could be detected in about $70 \%$ of samples from necrotic bone using histological techniques [15, 23-25]. In this study, we have detected Actinomyces species only in 12 of the 116 samples (10.3\%). This disparity might be due to the technical challenges in the
Fig. 4 High rate of penicillin resistance among gram-negative isolates. A Penicillin resistance was observed in $39 \%$ of grampositive and $78 \%$ of gramnegative genera, respectively. Of all isolates tested, $61 \%$ were found to be resistant against penicillin, and in $70 \%$ of all samples, at least one resistant species was detected. b Overview over initial antibiotic regimen in MRONJ patients; amoxicillin and clavulanic acid were administered twice daily; oral moxifloxacin was used in a once-daily regimen a

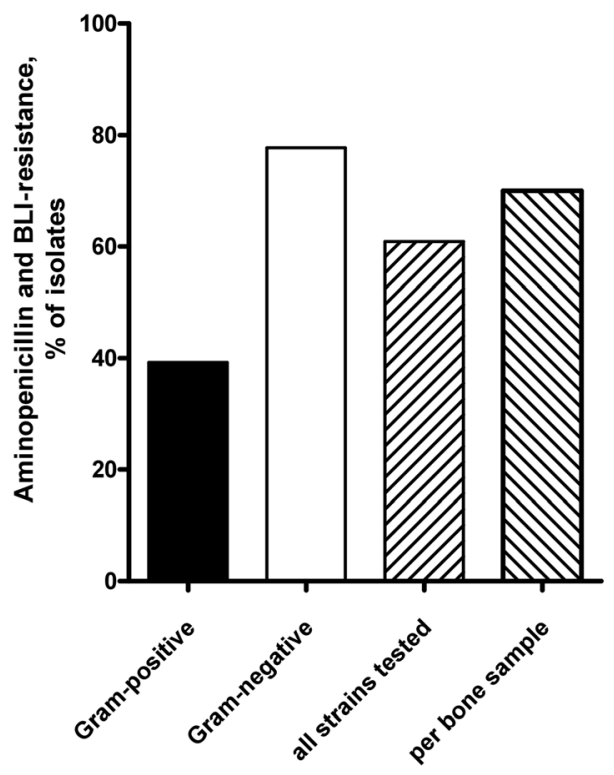

b

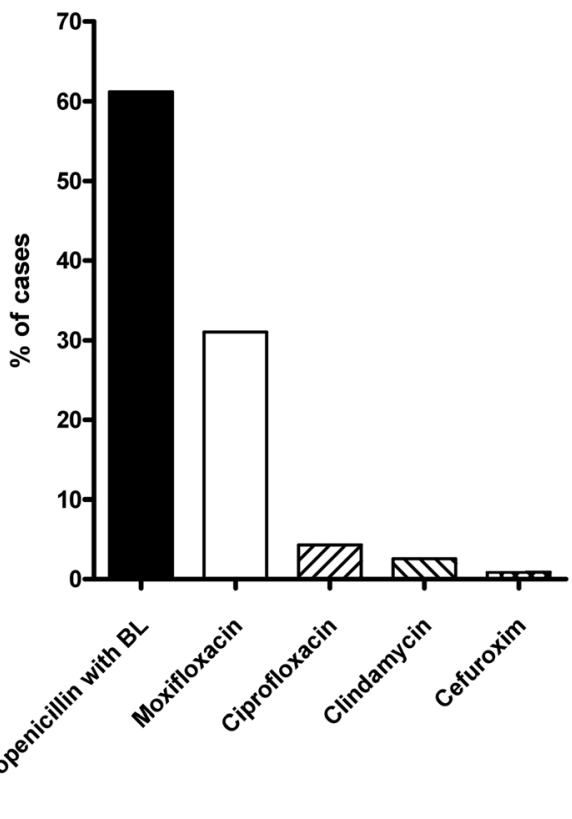


Fig. 5 Influence of susceptibility testing on antibiotic treatment in MRONJ patients. Results from susceptibility testing were available for 52 of 116 cases. This led to changes in the antibiotic treatment in 22 of the 52 patients, whereas the treatment was continued in 16 patients. In 8 patients, although the presence of resistant bacteria was detected, no changes to the antibiotic treatment were made (e.g., because results from the susceptibility testing were overlooked). In one patient treated with moxifloxacin and the presence of resistance, the treatment was discontinued. In 5 patients, the antibiotic regimen was changed for other reasons, e.g., intolerance

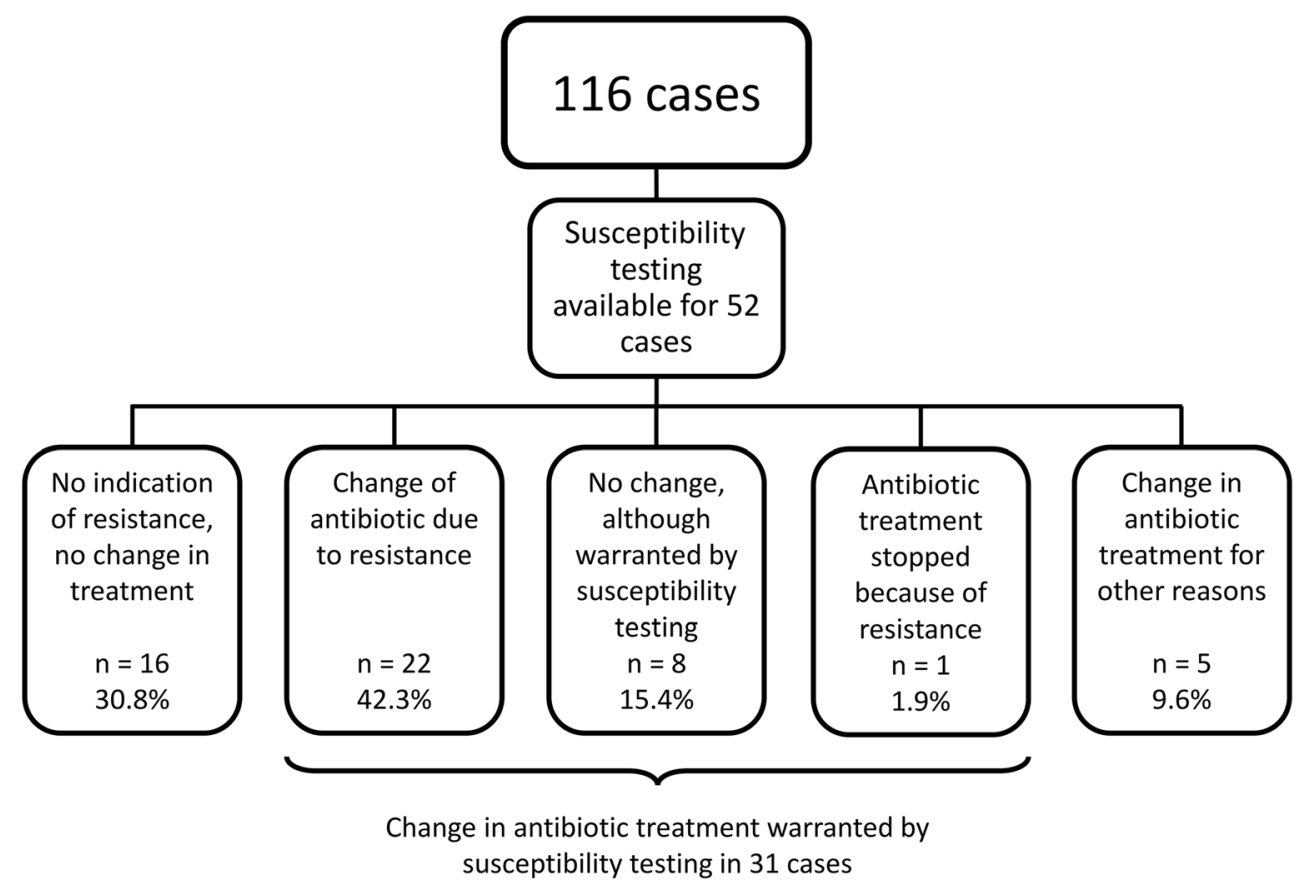

morbidities. Using routine susceptibility testing may help to avoid using fluoroquinolones in cases where it may not be necessary.

\section{Conclusion}

If antibiotic treatment is warranted in MRONJ patients, the empiric choice of antibiotics should consider the high rate of gram-negative bacteria, or cultivation methods should be used to help guide the antibiotic treatment. The common application of antibiotics, especially clindamycin or amoxicillin in dental or oral surgical procedures, may lead to an increasing frequency of bacterial resistance. This can become a serious problem, especially in patients with MRONJ, osteoradionecrosis, or other infectious diseases of the bone. Therefore, antibiotic treatment should be reconsidered in each case and each patient. Especially in MRONJ patients, an effective antibiotic therapy might reduce the risk for wound healing disorders resulting in recurrence of necrotic bone areas. Therefore, further research is warranted for the evaluation and development of potentially more rational antibiotic therapies, with a special emphasis on the efficient delivery of antibiotics to the hypovascular bone matrix.

Funding Open Access funding enabled and organized by Projekt DEAL. 


\section{Compliance with ethical standards}

Conflict of interest The authors declare that they have no conflict of interest.

Ethical approval Ethical approval was waived by the local Ethics Committee of University Hamburg Medical Center in view of the retrospective nature of the study and all the procedures being performed were part of the routine care.

Informed consent Informed consent was obtained from all individual participants included in the study.

Open Access This article is licensed under a Creative Commons Attribution 4.0 International License, which permits use, sharing, adaptation, distribution and reproduction in any medium or format, as long as you give appropriate credit to the original author(s) and the source, provide a link to the Creative Commons licence, and indicate if changes were made. The images or other third party material in this article are included in the article's Creative Commons licence, unless indicated otherwise in a credit line to the material. If material is not included in the article's Creative Commons licence and your intended use is not permitted by statutory regulation or exceeds the permitted use, you will need to obtain permission directly from the copyright holder. To view a copy of this licence, visit http://creativecommons.org/licenses/by/4.0/.

\section{References}

1. Assaf AT, Smeets R, Riecke B, Weise E, Grobe A, Blessmann M, Steiner T, Wikner J, Friedrich RE, Heiland M et al (2013) Incidence of bisphosphonate-related osteonecrosis of the jaw in consideration of primary diseases and concomitant therapies. Anticancer Res 33:3917-3924

2. Chan BH, Yee R, Puvanendran R, Ang SB (2018) Medicationrelated osteonecrosis of the jaw in osteoporotic patients: prevention and management. Singapore Med J 59:70-75

3. Bilezikian JP (2006) Osteonecrosis of the jaw-do bisphosphonates pose a risk? N Engl J Med 355:2278-2281

4. Diniz-Freitas M, Fernandez-Feijoo J, Diz Dios P, Pousa X, Limeres J (2018) Denosumab-related osteonecrosis of the jaw following non-surgical periodontal therapy: a case report. J Clin Periodontol 45:570-577

5. Compain H, Berquet A, Loison-Robert LS, Ahossi V, Zwetyenga $\mathrm{N}$ (2018) Duration of treatment with bisphosphonates at the time of osteonecrosis of the jaw onset in patients with rheumatoid arthritis. Review. J Stomatol Oral Maxillofac Surg 119:177-181

6. Owosho AA, Liang STY, Sax AZ, Wu K, Yom SK, Huryn JM, Estilo CL (2018) Medication-related osteonecrosis of the jaw: an update on the memorial Sloan Kettering cancer center experience and the role of premedication dental evaluation in prevention. Oral Surg Oral Med Oral Pathol Oral Radiol 125:440-445

7. De Antoni CC, Matsumoto MA, Silva AAD, Curi MM, Santiago Junior JF, Sassi LM, Cardoso CL (2018) Medication-related osteonecrosis of the jaw, osteoradionecrosis, and osteomyelitis: a comparative histopathological study. Braz Oral Res e23:32

8. Nicolatou-Galitis O, Migliorati C (2018) Osteonecrosis of the jaw $(\mathrm{ONJ})$ in patients who receive bone targeting agents (BTAs): the power of e-learning. Ecancermedicalscience 12:ed77

9. Ruggiero SL, Mehrotra B (2009) Bisphosphonate-related osteonecrosis of the jaw: diagnosis, prevention, and management. Annu Rev Med 60:85-96

10. Galis B, Zajko J, Hirjak D, Vanko L, Kupcova I, Jurkemik J, Gengelova P, Mikuskova K, Halmova K, Riznic M, Czako L
(2017) Is the prevalence of the medication-related osteonecrosis of the jaws underestimated, evaluation in oncological and nononcological disease. Bratisl Lek Listy 118:724-731

11. Ruggiero SL, Dodson TB, Fantasia J, Goodday R, Aghaloo T, Mehrotra B, O'Ryan F (2014) American Association of Oral and Maxillofacial Surgeons: American Association of Oral and Maxillofacial Surgeons position paper on medication-related osteonecrosis of the jaw-2014 update. J Oral Maxillofac Surg 72: 1938-1956

12. Baba A, Goto TK, Ojiri H, Takagiwa M, Hiraga C, Okamura M, Hasegawa S, Okuyama Y, Ogino N, Yamauchi H et al (2018) CT imaging features of antiresorptive agent-related osteonecrosis of the jaw/medication-related osteonecrosis of the jaw. Dentomaxillofac Radiol 47:20170323

13. Otto S, Pautke C, Van den Wyngaert T, Niepel D, Schiodt M (2018) Medication-related osteonecrosis of the jaw: prevention, diagnosis and management in patients with cancer and bone metastases. Cancer Treat Rev 69:177-187

14. Assaf AT, Zrnc TA, Remus CC, Adam G, Zustin J, Heiland M, Friedrich RE, Derlin T (2015) Intraindividual comparison of preoperative (99 m)Tc-MDP SPECT/CT and intraoperative and histopathological findings in patients with bisphosphonate- or denosumab-related osteonecrosis of the jaw. J Craniomaxillofac Surg 43:1461-1469

15. Boff RC, Salum FG, Figueiredo MA, Cherubini K (2014) Important aspects regarding the role of microorganisms in bisphosphonate-related osteonecrosis of the jaws. Arch Oral Biol 59:790-799

16. De Bruyn L, Coropciuc R, Coucke W, Politis C (2018) Microbial population changes in patients with medication-related osteonecrosis of the jaw treated with systemic antibiotics. Oral Surg Oral Med Oral Pathol Oral Radiol 125:268-275

17. Schwartz HC (2015) American Association of Oral and Maxillofacial Surgeons position paper on medication-related osteonecrosis of the jaw-2014 update and CTX. J Oral Maxillofac Surg 73:377

18. Yarom N, Shapiro CL, Peterson DE, Van Poznak CH, Bohlke K, Ruggiero SL, Migliorati CA, Khan A, Morrison A, Anderson H et al (2019) Medication-related osteonecrosis of the jaw: MASCC/ISOO/ ASCO Clinical Practice Guideline. J Clin Oncol 37:2270-2290

19. Pimolbutr K, Porter S, Fedele S (2018) Osteonecrosis of the jaw associated with antiangiogenics in antiresorptive-naive patient: a comprehensive review of the literature. Biomed Res Int 2018: 8071579

20. Montefusco V, Gay F, Spina F, Miceli R, Maniezzo M, Teresa Ambrosini M, Farina L, Piva S, Palumbo A, Boccadoro M, Corradini P (2008) Antibiotic prophylaxis before dental procedures may reduce the incidence of osteonecrosis of the jaw in patients with multiple myeloma treated with bisphosphonates. Leuk Lymphoma 49:2156-2162

21. Schiegnitz E, Al-Nawas, B, Hoefert, S, Otto, S, Pautke, C, Ristow, O, Voss, P, Grötz, KA: S3-Leitlinie Antiresorptiva-assoziierte Kiefernekrosen (AR-ONJ). 2012

22. Socransky SS, Haffajee AD, Cugini MA, Smith C, Kent RL Jr (1998) Microbial complexes in subgingival plaque. J Clin Periodontol 25:134-144

23. Panya S, Fliefel R, Probst F, Troltzsch M, Ehrenfeld M, Schubert S, Otto S (2017) Role of microbiological culture and polymerase chain reaction (PCR) of actinomyces in medication-related osteonecrosis of the jaw (MRONJ). J Craniomaxillofac Surg 45:357-363

24. Hansen T, Kunkel M, Weber A, James Kirkpatrick C (2006) Osteonecrosis of the jaws in patients treated with bisphosphonates histomorphologic analysis in comparison with infected osteoradionecrosis. J Oral Pathol Med 35:155-160

25. Jacobsen C, Metzler P, Obwegeser JA, Zemann W, Graetz KW (2012) Osteopathology of the jaw associated with bone resorption 
inhibitors: what have we learned in the last 8 years? Swiss Med Wkly 142:w13605

26. Kononen E, Wade WG (2015) Actinomyces and related organisms in human infections. Clin Microbiol Rev 28:419-442

27. Walker GT, Quan J, Higgins SG, Toraskar N, Chang W, Saeed A, Sapiro V, Pitzer K, Whitfield N, Lopansri BK et al (2019)
Predicting antibiotic resistance in gram-negative bacilli from resistance genes. Antimicrob Agents Chemother 63(4):e02462-18

Publisher's note Springer Nature remains neutral with regard to jurisdictional claims in published maps and institutional affiliations. 